\title{
Infrared modeling of the starburst clone NGC 3603
}

\author{
Daniel Devost ${ }^{1,2}$ and Claus Leitherer ${ }^{1}$ \\ ${ }^{1}$ Space Telescope Science Institute ${ }^{3}$, \\ 3700 San Martin Drive, Baltimore, MD 21211, USA \\ ${ }^{2}$ Département de physique and Observatoire du mont Mégantic, \\ Université Laval, Québec, P.Q. G1K 7P4, Canada
}

\begin{abstract}
We present results of an HST archive study of the star cluster NGC 3603. The color-magnitude diagram (CMD) and the radial profiles have been derived using HST-NICMOS F110W and F171M images. As expected, the CMD shows that the cluster is very young $(<3 \mathrm{Myr})$ and that a significant portion of the stellar population $\left(M<4 \mathrm{M}_{\odot}\right)$ is on the pre-main sequence phase. From the radial profiles, we derive a similar half-light radius for both wavebands of $0.17 \mathrm{pc}$.
\end{abstract}

\section{Introduction}

Starburst galaxies are the dominant extragalactic population in the local universe at luminosities above $10^{11} \mathrm{~L} \odot$. They are powered by young stars which are formed in numerous compact (sizes of a few pc), massive (masses of $10^{5-6} \mathrm{M}_{\odot}$ ) star clusters. Lack of spatial resolution and severe dust extinction preclude a detailed analysis of the stars and gas in the clusters.

Although not quite as luminous as a typical starburst cluster, NGC 3603 is considered a twin of 30 Doradus in the LMC (Moffat et al. 1994). Several studies of 30 Doradus have led to substantial insight into the physics of starbursts in metal-poor, dust-free irregular galaxies (Chu \& Kennicutt 1994). NGC 3603 is at a distance of $7 \mathrm{kpc}$ and has an extinction of $E_{B-V}=1.44 \mathrm{mag}$ (Drissen et al. 1995). This allows a detailed analysis of its stellar and nebular properties.

\section{Analysis and future directions}

Figure 1a compares the theoretical isochrones obtained with the code of Leitherer et al. (1999), with the observed data points. This figure clearly shows that we are detecting stars down to $1 \mathrm{M}_{\odot}$ and that a pre-MS phase for stars with masses lower than $4 \mathrm{M}_{\odot}$ is present. Also, a well defined main sequence can be seen with an upper mass limit in the range of $25-30 \mathrm{M}_{\odot}$.

Figure 1b shows the radial profiles derived from both filters using a radial increment of $0.1 \mathrm{pc}$. The profiles were fitted with a Gaussian curve. The best fit to the data points gives a half-light radius of $0.17 \mathrm{pc}$ for both filters, a peak

\footnotetext{
${ }^{3}$ Operated by Aura, Inc., for NASA under contract NAS5-26555
} 

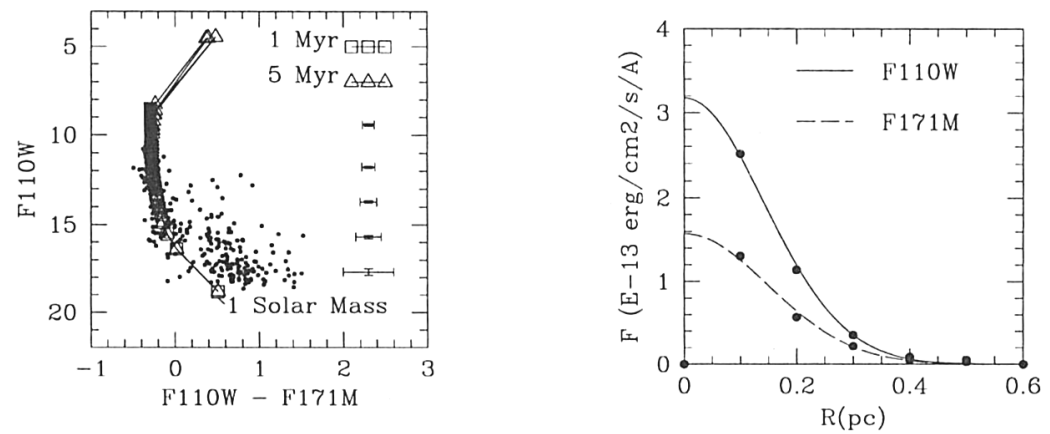

Figure 1. (a) Left panel: Color-magnitude diagram of the central $0.6 \mathrm{pc}$ of NGC 3603. The isochrones are for a population of stars at $1 \mathrm{Myr}$ (square) and $5 \mathrm{Myr}$ (triangle) of age and the symbols denote a $1 \mathrm{M}_{\odot}$ increment. All the stars in the models have solar metallicity and their masses are ranging from 1 to $120 \mathrm{M}_{\odot}$. (b) Right panel: Radial profile of NGC 3603 in the F110W and in the F171M filters. The half-light radius is $0.17 \mathrm{pc}$ in both filters.

central intensity of 3.17 and $1.57 \times 10^{-13} \mathrm{erg} \mathrm{cm}^{-2} \mathrm{~s}^{-1} \AA^{-1}$ for the $\mathrm{F} 110 \mathrm{~W}$ and the F171M, respectivly.

We can hope to achieve with NGC 3603 for dusty, metal-rich starbursts what has been achieved previously with 30 Doradus for dust-free, metal-poor objects. A detailed study is on the way to determine whether NGC 3603 can be considered as a good template for star clusters that belong to distant starbursting galaxies.

\section{References}

Moffat, A.F.J., Drissen, L., Shara, M.M. 1994, ApJ 436, 183

Chu, Y.-H., Kennicutt, R.C. 1994, in: J.E. Dyson \& E.B. Carling (eds.), Kinematics and Dynamics of Diffuse Astrophysical Media, ApSS 216, 253

Drissen, L., Moffat, A.F.J., Walborn, N.R., Shara, M.M. 1995, AJ 110, 2235

Leitherer, C., Schaerer, D., Goldader, J.D., González-Delgado, R.M., Robert, C., Kune, D.F., de Mello, D.F., Devost, D., Heckman, T.M. 1999, ApJ in press 